\title{
Delayed radiation necrosis of the central nervous system in patients irradiated for pituitary
}

\section{tumours}

\author{
Padraic J Grattan-Smith, John G Morris, Allan O Langlands
}

\begin{abstract}
Four cases of delayed radiation necrosis involving the CNS were found in a group of 46 patients irradiated for pituitary tumours over a six year period. This occurred in three of 11 patients with Cushing's disease representing an incidence of $27 \%$ in this group. There were no cases among 11 patients with acromegaly or among seven with prolactinomas. One case $(6 \%)$ was found in the 17 patients with chromophobe adenomas. Standard doses of radiation were delivered to these patients and the findings support suggestions that the metabolic disturbances of Cushing's disease may reduce tolerance to radiation. Our results and a literature review indicate that if radiotherapy is used to treat Cushing's disease, the total dose should be less than $50 \mathrm{~Gy}$ at $2 \mathrm{~Gy}$ per day fractionation.
\end{abstract}

$(\Im$ Neurol Neurosurg Psychiatry 1992;55:949-955)

Pituitary tumours are less aggressive than many CNS tumours but still pose formidable problems as management requires control both of their local destructive effects and of the remote metabolic disturbances created by pituitary hormone dysfunction. Current treatment includes surgery, radiotherapy and medical therapy each given alone or in combination. Radiotherapy was first used in $1909^{1}$ and remains one of the principal therapeutic options. Delayed cerebral radiation necrosis (DCRN) is a potential hazard, ${ }^{2-7}$ but is thought to be uncommon with modern techniques of pituitary radiation. There have been many recent reports recording the irradiation of large numbers of patients with a very low incidence of DCRN. ${ }^{8-13}$ Grossman et al, ${ }^{14}$ reported no complications in 332 patients irradiated for pituitary tumours in their institution over a 20 year period. Sheline, ${ }^{15}$ combining his own and Kramer's data accumulated 500 patients who had received radiation for pituitary adenomas without a single case of

Table 1 Details of the 46 patients reviewed in the study

\begin{tabular}{lrrrrrc}
\hline Tumour type & Number & $M$ & $F$ & Age & SSE & Surgery \\
\hline Cushing's D & 11 & 0 & 11 & 46 & 0 & 3 \\
Acromegaly & 11 & 8 & 3 & 59 & 4 & 4 \\
Prolactinomas & 7 & 1 & 6 & 44 & 3 & 5 \\
Chromophobe A & 17 & 13 & 4 & 55 & 17 & 16
\end{tabular}

$\mathbf{M}=$ male; $\mathbf{F}=$ female; $S S E$ = suprasellar extension of the tumour. Age = mean age in years; Surgery $=$ number of patients treated surgically.
DCRN. In contrast, a number of other studies have reported a substantial incidence of DCRN. ${ }^{16-23}$

The difference in the reported incidence of DCRN following pituitary radiation may have a number of explanations. Studies vary markedly in terms of total dose, dose per fraction and duration of radiotherapy. Follow-up tends to be dispersed among neurosurgeons, radiotherapists, neurologists and endocrinologists, each group viewing treatment outcome from a different perspective. A causal relationship between the neurological deficit and radiotherapy may not be recognised or may be difficult to establish as the peak incidence of DCRN is one to three years after radiation, ${ }^{4}$ but it may not appear until many years later. DCRN has to be distinguished from tumour recurrence, the empty sella syndrome and coincidental cerebrovascular disease. This was particularly difficult in the era before CT scanning. Reported series often encompassed a long time span and analysis was based on retrospective case note reviews. It is likely that the discrepancy in the reported incidence of DCRN is partly due to failure to detect all cases.

In this study we carefully examined a group of patients irradiated at Westmead Hospital using modern radiotherapy techniques to assess the prevalence of DCRN.

\section{Patients and methods}

Fifty one patients received radiotherapy for pituitary tumours at Westmead Hospital between January 1980 and December 1985. Four had gone overseas and could not be reviewed and another refused to be seen. Of the remaining 46 patients, the subjects of the study, there were 11 cases of Cushing's disease, 11 of acromegaly, 7 prolactinomas and 17 patients with chromophobe adenomas. Clinical details are listed in table 1 . Seven had died. In these cases, physicians who had cared for them were contacted to discover details of their final illness. All surviving patients were seen by a neurologist (either JM or PGS) in 1987 , at least one year after the completion of radiotherapy. During the review, the case notes were examined and a careful history was obtained from the patients and their relatives (if present) with particular emphasis on memory, behaviour changes, seizures, strokes or visual problems since the radiotherapy. A formal neurological examination followed with emphasis on visual field testing, both by confrontation and by testing for central desaturation with a red pin. 
Table 2 Distribution of cases according to diagnosis and radiation therapy technique

\begin{tabular}{llll}
\hline & Parallel pair & Arc & 3-field isocentric \\
\hline Cushing's D & 9 a & 1 & 1 \\
Acromegaly & 9 aaab & 2 & - \\
Prolactinoma & 6 aaa & 1 & 3 \\
Chromophobe A & 14 aaa & - & - \\
\hline
\end{tabular}

All cases CRE $=1500-1560$ reu, unless otherwise stated. $a=$ dose in range 1560-1660 reu; $\mathrm{b}=$ dose greater than $1660 \mathrm{reu}$.

\section{Radiotherapy}

All patients were immobilised in a plastic cast during treatment and a simulator or planning CT was used to determine the irradiated volume. Treatment was delivered using $6 \mathrm{Mev}$ photons given by a parallel pair in 38 patients, a 3 field technique in 4 patients and a rotational technique in 4 patients. In most cases $\mathrm{CRE}^{\star}$ was between 1500 and 1560 reu. Details of the radiation dosage are given in table 2 .

\section{Results}

Radiation necrosis

Four cases of DCRN were identified.

Case 1 A 55 year old woman with Cushing's disease was initially treated by a left adrenalectomy. There were many associated medical problems including pyoderma gangrenosa, ischaemic heart disease, congestive cardiac failure, Wolff-Parkinson-White syndrome, hypertension and hypothyroidism. A cranial CT scan was normal. Five years after diagnosis radiotherapy was given because of persistent hypercortisolism. She received 50 Gy to a $5 \times$ 5 centimetre field with a 120 degree arc rotation (figure 1). Individual fractions were 2 Gy. TDF was 82 and CRE 1550 reu. During the radiotherapy she received metyrapone to inhibit cortisol secretion and hydrocortisone as steroid replacement. Seventeen months later she developed confusion, seizures and a left

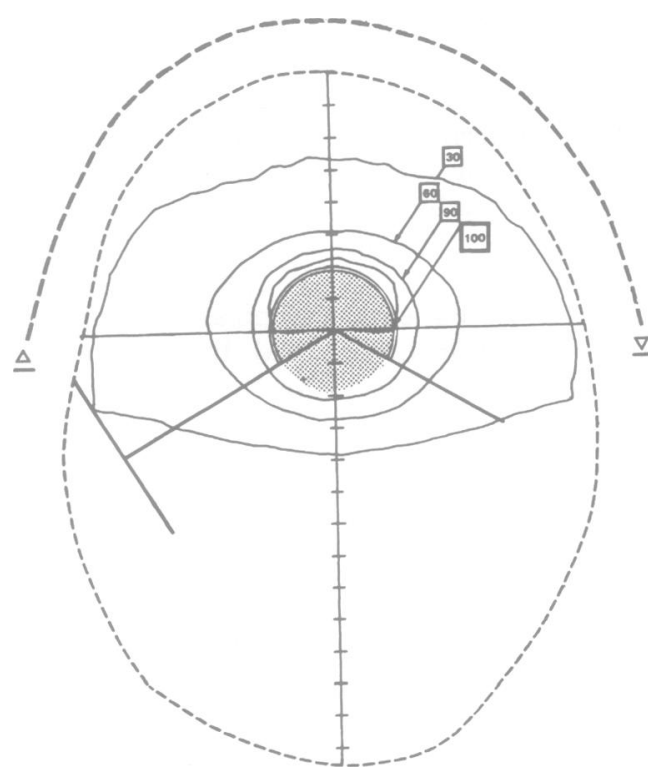

Figure 1 The patient received $50 \mathrm{~Gy}$ to a $5 \times 5 \mathrm{~cm}$ field with 120 degree arc rotation. Individual fractions were $2 G y$.

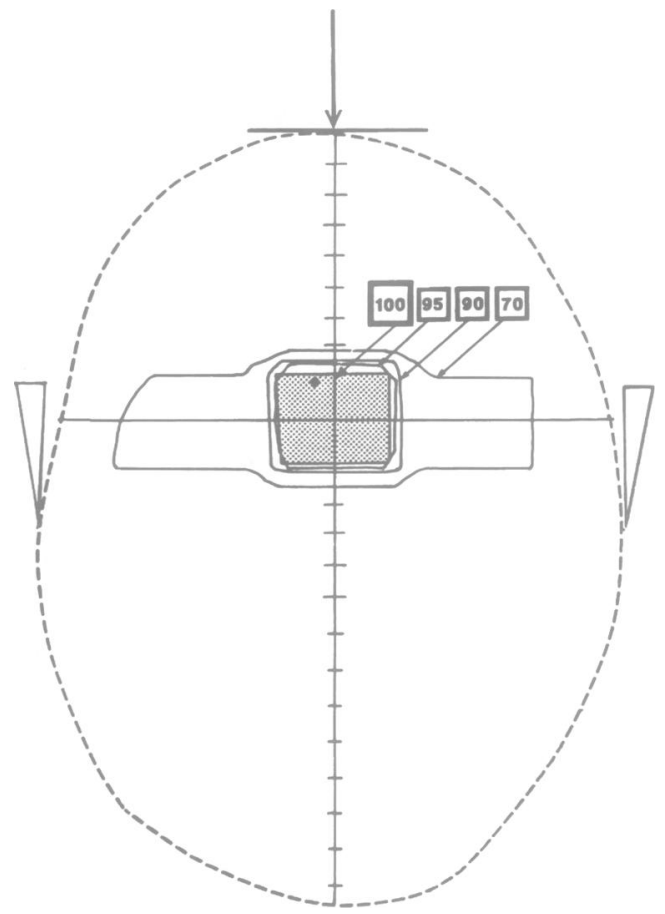

Figure 2 The patient received 50 Gy via a 3 field technique to a volume of 64 cubic centimetres with a daily fraction of $2 \cdot 1 \mathrm{~Gy}$.

hemiparesis. CT scan showed low density lesions in both internal capsules and a low density non-enhancing lesion in the left temporal lobe. She continued to deteriorate and was transferred to a nursing home. When reviewed there in 1987, she was demented and bedridden. The left hemiparesis was still evident and she had now developed blindness in her left eye with optic atrophy.

This patient had many risk factors for stroke and her initial problems 17 months post radiotherapy were attributed to cerebrovascular disease. The subsequent development of optic atrophy and the progressive nature of her illness, involvement of both cerebral hemispheres and fits are more readily attributable to radiation necrosis than cerebrovascular disease.

Case 2 A 52 year old woman was diagnosed with Cushing's disease after she had been treated for hypertension for the preceding five years. A cerebral CT scan was normal. Radiotherapy was given after a brief trial of aminoglutethamide and metyrapone. A total dose of 50 Gy was delivered using a 3 field technique to a volume of 64 cubic centimetres with a daily fraction of $2 \cdot 10 \mathrm{~Gy}$ (figure 2 ). TDF was 83 and CRE 1570 reu. During the radiotherapy she received metyrapone blockade with dexamethasone replacement. Eighteen months later, she suddenly lost the vision in her right eye. Initially there was a horizontal deficit with loss of the upper field but total blindness developed over a few days. Medications at the

${ }^{\star} \mathrm{CRE}=$ cumulative radiation effect. This is a simple notation to normalise different schedules and regimes as a dimensionless number. It represents a summation of the effects of radiation fractions or groups of fractions with account being taken of the injury caused by prior fractions. ${ }^{24}$ 


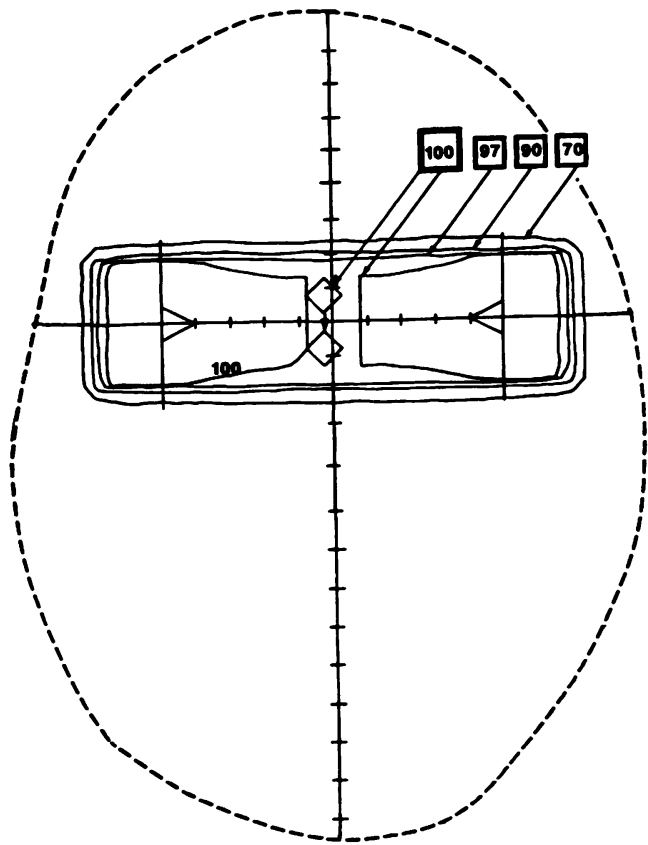

Figure 3 The patient received 50 Gy with a daily fraction of 2 Gy via $5 \times 5 \mathrm{~cm}$ parallel opposed fields.

time included aminoglutethamide, metyrapone and polaramine. When seen one month later, there was complete blindness in that eye due to optic atrophy. Visual acuity in her left eye was $6 / 15$. When questioned, she complained of loss of recent memory. Visual evoked responses were absent from the right eye and there was a normal response on the left. Erythrocyte sedimentation rate was 11 millimetres per hour. Cranial CT and MRI scans were normal.

Four months later she was admitted with failing vision in her left eye. She could no longer see television, read a book or distinguish faces. The visual acuity in the left eye had declined to $6 / 36$ and both optic discs were now pale. Her ESR remained normal. A course of oral prednisone produced no improvement. Her memory problems have persisted.

Case 3 A 61 year old woman presented with a five month history of headaches, difficulty climbing stairs and hypertension and was found to have Cushing's disease. A cerebral CT scan revealed a partially empty sella with a low density area within the inferior portion of the gland. She was treated with radiotherapy. The total dose was 50 Gy with a daily fraction of 2 Gy via $5 \times 5 \mathrm{~cm}$ parallel opposed fields (figure 3). TDF was 82 and CRE $1550 \mathrm{reu}$. During the radiotherapy she received metyrapone and dexamethasone.

Fifteen months later she noted slight blurring of the vision in her left eye. This slowly progressed over the next six months until she could only detect hand movements and then deteriorated no further. A cerebral CT scan was unchanged. In 1987, she had evidence of left optic atrophy and also admitted to a recent decline in short term memory although she felt this had never been good. Visual evoked responses were absent on full and half field stimulation of the left eye and were poorly

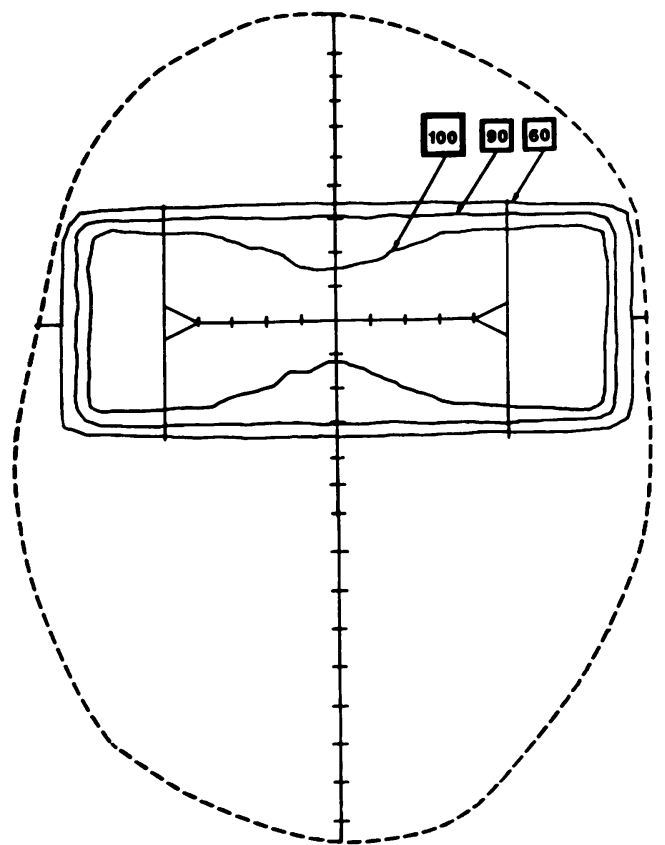

Figure 4 The patient received 50 Gy to $7 \times 7 \mathrm{~cm}$ parallel opposed fields with a daily fraction of $2 \cdot 17 \mathrm{~Gy}$.

formed on stimulation of the right eye. This suggested either chiasmal or bilateral optic nerve damage, worse on the left. Medications at the time of assessment included aminoglutethamide, metyrapone, dexamethasone, metoprolol, spironolactone, premarin, calcium gluconate and mylanta. A subsequent cerebral CT scan in 1989 revealed a slight decrease in the size of the gland but the chiasm in all scans has remained above the sella.

Case 4 This 61 year old man presented with bitemporal hemianopia. He was found to have a 3 centimetre pituitary tumour extending into the cavernous sinus and suprasellar regions. A chromophobe adenoma was incompletely removed through a right frontal craniotomy. In the immediate post-operative period, there was a haemorrhage in the region of the pituitary which was associated with generalised seizures. These were controlled by anti-convulsants. Hydrocephalus developed and a ventriculoperitoneal shunt was inserted. Radiotherapy started one month later. Repeat head CT scan showed the tumour continued to extend beyond the pituitary fossa. The patient had hypopituitarism and was receiving replacement dexamethasone. A midline dose of $50 \mathrm{~Gy}$ was applied in 23 daily fractions of $2 \cdot 17$ Gy to parallel pair fields of $7 \times 7 \mathrm{cms}$ (figure 4). TDF was 83 and CRE 1550 reu. Six months later the tumour recurred (figure 5a). Following further attempts at tumour removal, the patient sustained a right frontal lobe infarct (figure 5b). Fourteen months after the radiotherapy he became confused and three months later there was a sudden deterioration in his level of consciousness. He spent most of his time asleep, had no spontaneous speech and mumbled incomprehensively when questioned. $\mathrm{He}$ continued to deteriorate and CT scans over the next three months revealed progressive ventricular dilatation (not relieved 
Figure 5 Case 4.

Changes in CT scan; a) $A$ large pituitary tumour is evident; b) post-operative frontal infarction; $c-f$ ) Progressive bilateral low density changes starting 14 months after radiotherapy.

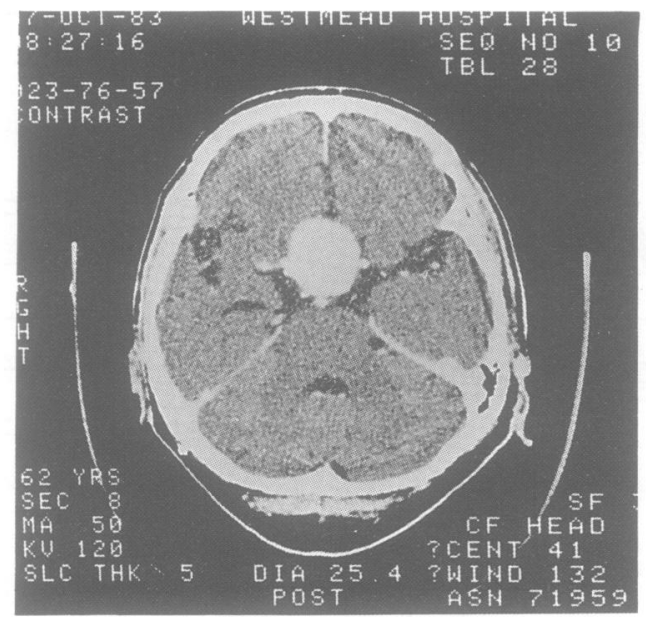

(a)

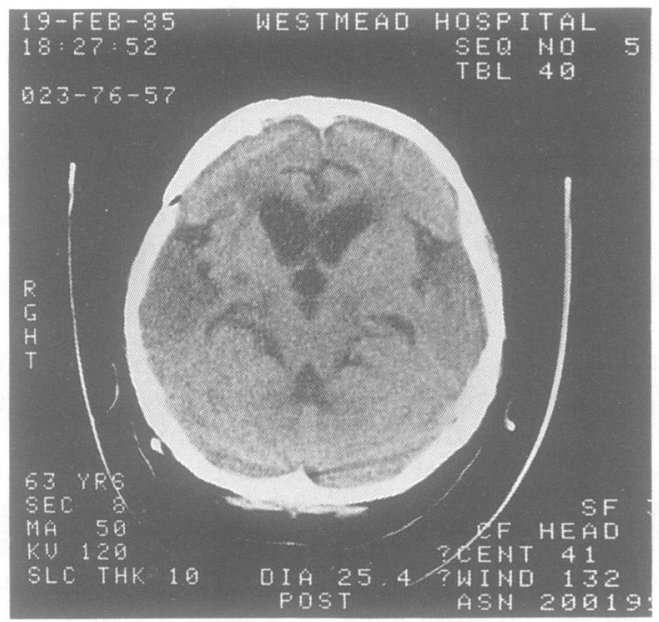

(c)

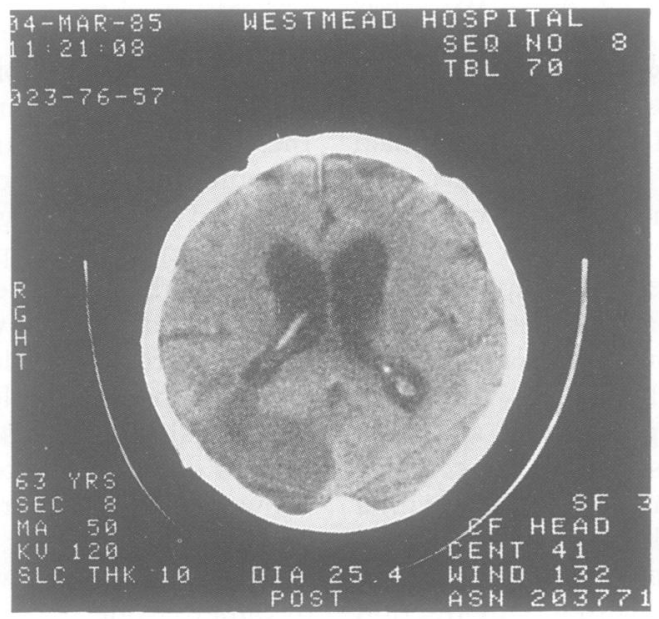

(e)

by further ventriculoperitoneal shunting) and the sequential appearance of low density lesions in the frontal, parietal, temporal and occipital lobes (figures 5-c, d, e, f). He subsequently died but necropsy examination was not performed.

The right frontal infarct probably resulted from the surgery. The subsequent progressive appearance of infarcts in the parietal and temporal lobes is typical of radiation necrosis. The likely cause of the occipital lesion was radiation damage to the posterior cerebral

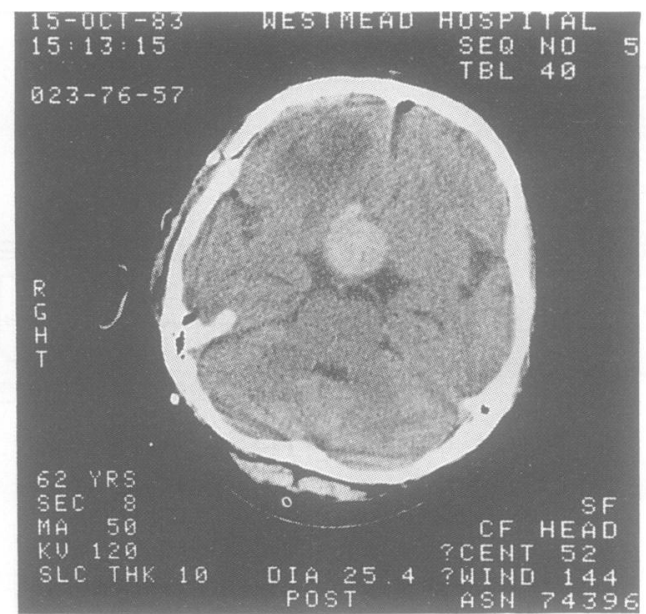

(b)

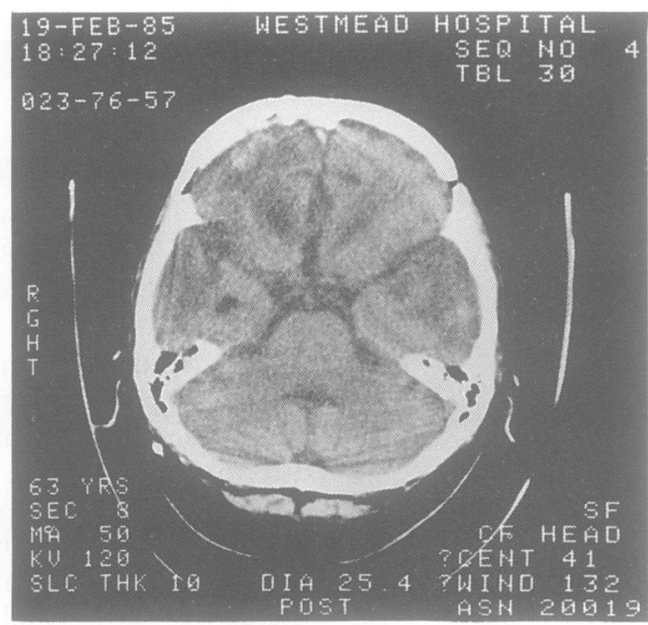

(d)

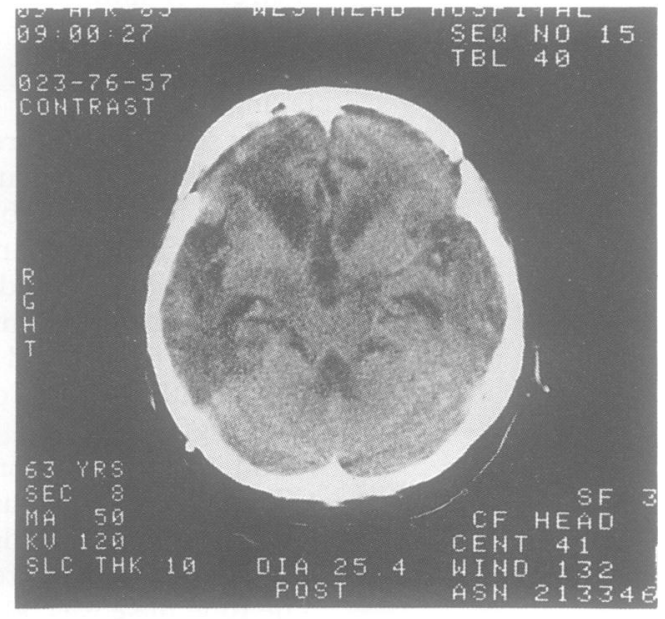

(f)

artery at its origin from the circle of Willis which is in the radiation field.

Details of the seven patients who died appear in table 3. One (case 4) was identified as a case of DCRN before his death. Most died in nursing homes and little information about their final illness is available. The only patient who had post-mortem neuropathological examination was patient 2 . This was performed at another institution. The pituitary was reported to be within normal limits and "old softening and degeneration" of the cerebrum 
Table 3 Cause of death of the seven patients who have died

\begin{tabular}{llll}
\hline Patient & Diagnosis & Age & Cause of death \\
\hline 1 & Cushing's D & 55 & Pneumonia \\
2 & Acromegaly & 54 & CCF, Cardiomyopathy \\
3 & Acromegaly & 54 & Metastatic breast CA \\
4 & Chromophobe adenoma & 86 & Cerebral thrombosis \\
5 & Chromophobe adenoma & 70 & Dementia \\
6 & Chromophobe adenoma & 38 & Metastatic Colon CA \\
7 & Chromophobe adenoma & 64 & DCRN \\
\hline
\end{tabular}

Age is in years. confirmation of the diagnosis. In cases 1-3, where the damage was primarily to the optic nerve or chiasm, the diagnosis has to rest on the clinical and radiological features as biopsy of an optic nerve would, in our view, be unethical. A biopsy of the left temporal lobe was not done in case 1 , as this would have involved subjecting a demented, bedridden patient to an invasive procedure to make the diagnosis of a condition for which there is no treatment. The lack of adequate necropsy information on the seven patients who died is a reflection of the dispersion of follow up between different specialists and general practitioners. There was no central, systematic monitoring of their progress and no concerted effort to obtain an adequate post-mortem neuropathological examination. This would appear to be a particular problem with pituitary tumours and is likely to have occurred in previous studies.

Aristizabel et al $^{16-18}$ have previously reported that the chiasm is particularly vulnerable to radiotherapy in Cushing's disease. They encountered five cases of DCRN amongst 122 patients with pituitary tumours. Four had optic pathway damage and one brainstem necrosis. DCRN occurred only in patients who had received a total dose of between $46 \mathrm{~Gy}$ and 52 $\mathrm{Gy}$, or individual fractions of more than $2 \mathrm{~Gy}$. In this sub-group three of nine patients with Cushing's disease, one of four with acromegaly and one of 13 with chromophobe adenomas developed DCRN. A literature review revealed that of 14 cases of DCRN in patients with pituitary tumours, who had received what the authors regarded as acceptable radiation doses, seven had Cushing's disease. Visual pathway damage was the most common problem, presenting either alone or in combination with cerebral damage. The authors postulated that the high circulating steroid levels present in Cushing's disease act as a radiosensitiser. It was also suggested that hypertension might have a role $\mathrm{r}^{2728}$ and that patients with acromegaly may be at increased risk.

Uncertainty still exists as to the optimum dose of radiotherapy for pituitary adenomas. Existing guidelines have evolved by inference from reports of complications attributed to excessive doses. Unfortunately in many studies there has been considerable variation in radiotherapy technique. In our study a parallel opposed pair of bitemporal fields was used in 38 of 46 patients, most receiving $50 \mathrm{~Gy}$ maximum in 25 fractions. Nevertheless, no firm conclusions can be drawn about the effect of technique on DCRN. Of the three patients with Cushing's disease who developed the syndrome, one had been treated by a parallel pair (one out of nine patients), one by an arc and one by a 3 field technique. These last two cases were the only patients in which these respective techniques were used.

Other factors which may have important modifying influences on the development of DCRN such as surgery, metabolic disturbances and coexistent medical problems in the patient have often not been considered. To compare different treatment schedules taking 
into account the interdependent biological effects of total dose, dose per fraction and overall treatment time, the time dose factor (TDF), a single numerical value can be used. Most current text-books suggest that a dose of $50 \mathrm{~Gy}$ in 25 fractions (TDF = 82) is safe for pituitary radiation. ${ }^{29}$

Optic pathway damage following pituitary radiation is not restricted to Cushing's disease. Harris and Levene ${ }^{21}$ described five cases of optic nerve damage among 55 patients with pituitary tumours; four had either chromophobe adenomas or craniopharyngiomas. It only occurred in the 28 patients who received $2.5 \mathrm{~Gy}$ or more per fraction. TDF ranged from 79 to 126 . The total dose which ranged from $40 \mathrm{~Gy}$ to as high as $70 \mathrm{~Gy}$ did not seem to be an important determinant of damage. Atkinson $e t a^{19}$ reported four cases of visual failure in 23 patients with acromegaly, one of whom also suffered frontal lobe and bony necrosis. The maximum dose was $45 \mathrm{~Gy}$, but each received a fraction of $2.8 \mathrm{~Gy}$ or greater. TDF ranged from 84 to 99 . Hammer ${ }^{20}$ described four cases of optic chiasmal radionecrosis, three of the patients having acromegaly and the other a chromophobe adenoma. Three received a total of $42.5 \mathrm{~Gy}$ in $2.8 \mathrm{~Gy}$ fractions (TDF $=83$ ) and one $42.5 \mathrm{~Gy}$ in $2 \cdot 1 \mathrm{~Gy}$ fractions $(T D F=71)$. The four patients presented over an 18 month period. In the preceding 10 years 83 other patients had been irradiated with the same technique and doses without incident. In contrast, Feek et al ${ }^{9}$ reported no adverse effects in the treatment of acromegaly using a fraction of $2.5 \mathrm{~Gy}$ but to a total dose of $37 \cdot 5 \mathrm{~Gy}$ (TDF=70).

The TDF of the five patients reported by Aristizabel et al ranged from 81-90. Large recent studies ${ }^{3031}$ with a significant incidence of DCRN do not give full details of the radiotherapy for specific cases and similar calculations cannot be made. TDF in our three patients with Cushing's disease and optic nerve damage was $82(\mathrm{~PB}, \mathrm{NC})$ and 83 (NC), well within the bounds of currently recommended doses. None had surgery as a possible predisposing factor. We feel this supports the suggestion of Aristizabel et al $^{16-18}$ of reduced radiation tolerance in this condition.

Because of the problems with Cushing's Disease patients, this group was looked at in more detail. TDF in all but patient NC was 82 . Of the 10 surviving patients, at follow up four were no longer receiving corticosteroid replacement or suppressive therapy, two had had bilateral adrenalectomy for persistent hypercortisolism, two developed hypopituitarism and one had persistent hypercortisolism. One patient had bilateral adrenalectomy before radiotherapy. Each patient with Cushing's disease and DCRN received metyrapone to block cortisol secretion and had replacement doses of steroids during their radiotherapy. We do not think the metyrapone had a role in their subsequent problems as five other patients were similarly treated and Ross $e t a l^{32}$ reported 14 patients irradiated for Cushing's disease who received metyrapone and aminoglutethamide without complications. In their study, a mean total dose of $46 \mathrm{~Gy}$ was delivered over 31 days. (One patient had retrobulbar neuritis but it is not clear if this occurred following radiotherapy).

There is additional evidence that steroid hormones may act as radiosensitisers. The cylindrical epithelium of the corpus uteri, normally resistant to radiation, becomes significantly more responsive when pretreated with medroxyprogesterone, both in vivo ${ }^{33}$ and in vitro. ${ }^{34}$ In a trial of adjuvant therapy for breast cancer, seven of 13 patients treated with radiation and medroxyprogesterone developed radiation induced oesophagitis and/or pneumonitis whereas this occurred in only one of nine treated with the same doses of radiation alone $(p<0.05) .^{35}$

There was one other patient in our study who developed DCRN. He had a large, very aggressive chromophobe adenoma. Three attempts at surgical removal were unsuccessful and each was associated with complications. This may well have predisposed to the subsequent development of DCRN. In any case, it is most unlikely he would have survived had radiotherapy not been given and DCRN must be seen here as an acceptable risk in the context of a desperate situation. Of the other six patients who died, the diagnosis of DCRN was considered in one but the evidence is not strong. There does not seem to be a "hidden" incidence of DCRN in this group.

In conclusion, we feel it is important to consider the type of tumour when irradiating pituitary adenomas. The metabolic disturbances of Cushing's disease pose different problems to the local destruction of a chromophobe adenoma and may produce an altered biological response to radiation. There has been a tendency to deliver similar doses of radiation irrespective of the patients condition or tumour type. Therapy should be individualised according to the patient's problems. An incidence of optic pathway damage of $27 \%$ is unacceptable and we feel that the total dose should be reduced to less than $50 \mathrm{~Gy}$ in Cushing's disease but cannot from this data determine a "safe" dose.

We thank Dr David Chipps for reviewing the manuscript.

1 Béclère $A$. The radiotherapeutic treatment of tumors of the hypophysis, gigantism and acromegaly. Arch Roentgenol Radiol 1909;14:147-50.

2 Crompton MR, Layton DD. Delayed radionecrosis of the brain following therapeutic $x$-radiation of the pituitary. Brain 1961;84:85-101.

3 Fukamachi A, Wakao T, Akai J. Brain stem necrosis after irradiation of pituitary adenoma. Surg N'iurol 1982 18:343-0.

4 Kramer $S$. The hazards of therapeutic irradiation of the

central nervous system. Clin Neurosurg 1968;15:301-19. radionecrosis: incidence and risk in relation to dose, time, radionecrosis: incidence and risk in relation to dose, time, Phys 1981;7:243-52.

6 Martins AN, Johnston JS, Henry JM, et al. Delayed radiation necrosis of the brain. $\mathcal{f}$ Ne'urosurg 1977 ; 47:336-45.

7 Pennybacker J, Russell DS. Necrosis of the brain due to radiation therapy. $\mathcal{F}$ Neurol Neurosurg Psychiatry 1948; 11:183-98.

8 Eastman RC, Gorden P, Roth J. Conventional supervoltage irradiation is an effective treatment for acromegaly. $f$ Clin Endocrinol and Metab 1979;48:931-40

9 Feek CM, McLelland J, Seth J, et al. How effective is external pituitary irradiation for growth hormone secreting pituitary tumours? Clin Endocrinologv 1984;20: 10 Mead KW. High Dose Radiotherapy for Pituitary Tumours. 
Aust Radiol 1981;25:229-36

11 Orth DN, Liddle GW. Results of treatment in 108 patients with Cushing's Syndrome. NEFM 1971;285:243-7.

12 Pistenma DA, Goffinet DR, Bagshaw MA, et al. Treatment of chromophobe adenomas with megavoltage irradiation. Cancer 1975;35:1574-82.

13 Salmi J, Grahne B, Valtonen S, Pelkonen R. Recurrence of chromophobe pituitary adenomas after operation and chromophobe pituitary adenomas after operation and 66:681-9.

14 Grossman A, Cohen BL, Charlesworth M, et al. Treatment of prolactinomas with megavoltage radiotherapy. Brit Med f 1984;288:1105-9.

15 Sheline GE, Wara WM, Smith V. Therapeutic irradiation and brain injury. Int $\mathcal{f}$ Radiation Oncology Biol Phys 1980;6:1215-28.

16 Aristizabal SA, Caldwell WL, Avila J, Mayer EG. Relationship of time dose factors to tumor control and complications in the treatment of Cushing's Disease by irradiation. Int $\mathcal{f}$ Radiation Oncology Biol Phys 1977;2:47-54.

17 Aristizabal SA, Caldwell WL, Avila J. The relationship of time-dose fractionation factors to complications in the treatment of pituitary tumors by irradiation. Int $\mathcal{f}$ Radiation Oncology Biol Phys 1977;2:667-73.

18 Aristizabal SA, Boone ML, Laguna JF. Endocrine factors influencing radiation injury to central nervous tissue. Int $\mathfrak{f}$ influencing radiation injury to central nervous

19 Atkinson AB, Allen IV, Gordon DS, et al. Progressive visual failure in acromegaly following external pituitary irradiation. Clin Endocrinology 1979;10:469-79.

20 Hammer HM. Optic chiasmal radionecrosis. Trans Ophthal Soc UK 1983;103:208-11.

21 Harris JR, Levene MB. Visual complications following irradiation for pituitary adenomas and craniopharyngio mas. Radiology 1976;120:167-71.

22 Mikhael MA. Dosimetric considerations in the diagnosis of radiation necrosis of the brain. In: Gilbert HA, Kagan $\mathrm{AR}$, eds. Radiation damage to the nervous . system. New York: Raven Press, 1980:59-91.
23 Peck FC, McGovern ER. Radiation necrosis of the brain in acromegaly. I Neurosurg 1966;25:536-42.

24 Andrews JR. The radiobiology of human cancer radiotherapy, 2nd ed. Baltimore: University Park Press, 1978:365-6.

25 Boghen D, Glaser JS. Ischaemic optic neuropathy. Brain 1975;98:689-708.

26 Plotz CM, Knowlton AI, Ragan C. The natural history of Cushing's Syndrome. Amer $\mathcal{F}$ Med 1952;13:597-614.

27 Asscher AW, Anson SG. Arterial hypertension and irradiation damage to the nervous system. Lancet 1962;2:
ton tion dam

28 Hopewell BJ, Wright EA. The nature of latent cerebral irradiation damage and its modification by hypertension. Br F Radiol 1970;43:161-7.

29 Mead KW. Precision pencil beam radiation therapy for pituitary adenomas. In: Withers HR, Peters LJ, eds. Innovations in radiation oncology. Berlin: Springer-Verlag, 1988:155-66.

30 Chun M, Masko GB, Hetelekidis S. Radiotherapy in the treatment of pituitary adenomas. Int $\mathcal{f}$ Radiation Oncology Biol Phys 1988;15:305-9.

31 Grigsby PW, Stokes S, Marks JE, Simpson JR. Prognostic factors and results of radiotherapy alone in the management of pituitary adenomas. Int $\mathcal{f}$ Radiation Oncology Biol Phys 1988;15:1103-10.

32 Ross WM, Evered DC, Hunter P, et al. Treatment of Cushing's Disease with adrenal blocking drugs and megavoltage therapy to the pituitary. Clin Radiol 1979;30:149-53.

33 Bonte I, Decoster JM, Ide P. Radiosensitisation of endometrial adenocarcinoma by means of medroxyprogesterone. Cancer 1970;25:907-10.

34 Huber $H$, Husslein $P$, Michalica $W$, Wagenbichler $P$. Radiosensitizing effect of medroxyprogesterone acetate on endometrial cancer cells in vitro. Cancer 1984; 54:999-1001.

35 De Greve J, Warson F, Deleu D, Storme G. Fatal pulmonary toxicity by the association of radiotherapy and medroxyprogesterone acetate. Cancer 1985;56:2434-6. 